\title{
Demonstration of a Sub-Pixel Outdoor Optical Camera Communication Link
}

\author{
Vicente Matus, Victor Guerra, Cristo Jurado-Verdu, Jose Rabadan, and Rafael Perez-Jimenez
}

\begin{abstract}
Optical Camera Communication (OCC) is an Optical Wireless Communication (OWC) technology that relies on general-purpose cameras to perform not only image-related tasks but also to receive data from optical sources. In general terms, OCC has been extensively studied in indoor scenarios and can be a competent and cost-effective alternative solution for wireless data transmission in Smart Cities for medium to long links up to hundreds of meters. In this paper, the feasibility of establishing an outdoor sub-pixel communication link is demonstrated. In this experiment, single $5 \mathrm{~mm}$ white LED transmitters located at distances of 90 and $130 \mathrm{~m}$ send simultaneous optical codes to a receiver based on a commercial camera, achieving a signal-tonoise ratio of $20 \mathrm{~dB}$ and $13 \mathrm{~dB}$, respectively. This work shows that although the geometrical projection within the transmitter's image is less than the pixel area, it is still possible to establish an effective communication link, with 8 bps per transmitter. At $130 \mathbf{~ m}$, the best performance was an error rate of $7,2 \cdot 10^{-3}$, and for $90 \mathrm{~m}$, no errors were detected.
\end{abstract}

Index Terms-Optical Camera Communication, Outdoor Optical Communication, Visible Light Communication.

\section{INTRODUCCIÓN}

$\mathbf{L}$ as comunicaciones ópticas usando cámaras $(\mathrm{OCC}-\mathrm{Op}$ tical Camera Communications) representan uno de los campos de investigación de mayor proyección dentro de las comunicaciones ópticas por luz visible (VLC - Visible Light Communications). Esta tecnología cuenta con un gran potencial para formar parte de la evolución de las nuevas redes Beyond 5G. En primer lugar, porque suponen un coste de integración muy bajo al utilizar tanto las cámaras fotográficas que actualmente están masivamente integradas en multitud de dispositivos finales de usuario (tales como smartphones, cámaras de vigilancia, dashcam, entre otros), como la tecnología led, que está ampliamente difundida por su bajo consumo y alta durabilidad.

Fruto de esta versatilidad, las OCC han sido incorporadas en el estándar IEEE 802.15.7r1 [1], donde se contemplan las dos estrategias fundamentales que se utilizan para el desarrollo de estos sistemas, y que varía en función de la técnica de adquisición de imágenes por parte de la cámara. Los sistemas Global Shutter (GS) y Rolling Shutter (RS). Los primeros utilizan principalmente sensores Charge-Coupled Device (CCD) que a la hora de adquirir una nueva imagen exponen todos sus píxeles de forma simultánea. Por el contrario, los sistemas Rolling Shutter (RS) basados principalmente en la tecnología

V. Matus, V. Guerra, C. Jurado-Verdu, J. Rabadan and R. Perez-Jimenez are with the Institute for Technological Development and Innovation in Communications, Universidad de Las Palmas de Gran Canaria, Spain.

Corresponding author: V. Matus, vmatus@idetic.eu.

This project has received funding from the European Union's Horizon 2020 research and innovation programme under the Marie Skłodowska-Curie grant agreement No 764461 . de transistores Complementary Metal-Oxide-Semiconductor (CMOS), realizan el escaneo de la imagen línea por línea de píxeles, de forma secuencial con un ligero solapamiento.

En escenarios interiores (oficinas, hogares), la aplicación más destacada para los sistemas OCC a día de hoy es la de posicionamiento por luz visible (VLP - Visible Light Positioning), en la que se combina la transmisión de datos y el procesado de imágenes para reconocer la geometría del entorno y vigilar las interacciones entre nodos móviles. Otras aplicaciones de interés son la monitorización inalámbrica de pacientes en hospitales, o en otras instalaciones donde el uso de señales de radiofrecuencia (RF) puede interferir el correcto desempeño de la instrumentación; y el intercambio de datos peer-to-peer $(\mathrm{P} 2 \mathrm{P})$ mediante balizas ópticas como alternativa a las comunicaciones de campo cercano (NFC - Near Field Communications) [2].

Cuando se plantea el uso de OCC en escenarios exteriores para aplicaciones de Smart Cities y control de redes de sensores, hay que tener en cuenta la presencia de fenómenos atmosféricos adversos no controlables tales como precipitaciones de lluvia y/o nieve, turbulencias inducidas por calor, presencia de partículas pequeñas en suspensión (aerosoles tales como vapor de agua, agentes contaminantes, polvo, etc.). Estos fenómenos producen tanto la atenuación como la dispersión temporal de la señal en la dirección del enlace, aumentando la tasa de error en la comunicación. Para hacer frente a estas condiciones se han propuesto distintas soluciones que se pueden dividir entre aquellas que alteran la óptica de la cámara, y las que ajustan de forma efectiva los parámetros internos de configuración del sensor de imagen.

En [3] se validó experimentalmente un enlace en exteriores de aproximadamente $400 \mathrm{~m}$, que hace uso del desenfoque óptico de la cámara, lo que permitió extender la superficie del led emisor e incrementar la tasa de transferencia hasta los 450 bps (bits por segundo). En los experimentos de [4] y [5] se muestran otros ejemplos en los que se compensa el efecto del canal mediante el uso de lentes de magnificación. El primer trabajo, se centró en una aplicación para un entorno vehicular basado en fotodiodos (PD - photo-diode) en lugar de cámaras, consiguiendo una distancia de enlace de $40 \mathrm{~m}$ condiciones climáticas ideales. En el segundo artículo se utilizó una cámara GS para establecer un enlace con un cartel luminoso situado a una distancia de $328 \mathrm{~m}$ con una tasa de transmisión efectiva de 15 bps con un $4 \%$ de error. Por último, en [6] se demuestra el uso de una lente de Fresnel para el establecimiento de un enlace vehicular VLC en un entorno emulado en laboratorio. En otros trabajos previos, [7], [8] se demuestra que el deterioro en la señal producido por la atenuación debida a fenómenos de turbulencia y neblina se pueden mitigar modificando la 
ganancia analógica del sensor de imagen sin necesidad de alterar la óptica de la cámara.

Sin embargo, en todos estos trabajos anteriormente mencionados se parte de la premisa de que el área proyectada de la fuente luminosa en la imagen afecta un número alto de píxeles, lo que quiere decir, que la proyección geométrica de la lámpara ocupa una porción destacable en la imagen [9], [10]. Esta consideración previa repercute en la distancia máxima de enlace, haciendo que o bien se necesiten superficies luminosas de área muy extensa o el uso de lentes telescópicas lo cual se traduce en una reducción del campo de visión y, por tanto, una penalización en las capacidades de monitorización simultánea de dispositivos.

En este trabajo se demuestra la viabilidad de un enlace GS para exteriores en el que la comunicación sucede a nivel de sub-píxel. El término sub-píxel hace referencia a que el área de proyección de la fuente es menor al área de un píxel. En una discusión preliminar podría asumirse la no viabilidad del enlace en base a que la luz emitida incide únicamente en un píxel. Sin embargo, en este trabajo se demuestra que la energía emitida por el LED afecta a los píxeles contiguos de forma que la señal puede ser recuperada con éxito.

Este artículo se estructura como sigue. El estado del arte en el campo de investigación de OCCse resume en la Sección II. La sección III explica en detalle el modelo de canal OCC y la nueva definición propuesta en este trabajo respecto a los escenarios sub-píxel. La sección IV presenta el diseño experimental y las métricas de interés, mientras que la sección V muestra los resultados obtenidos. Finalmente, en la sección VI se extraen las conclusiones y el potencial impacto de los hallazgos de este trabajo.

\section{EstAdo DEL ARTE}

La rama de OCC surge a partir de un subcampo de las comunicaciones ópticas inalámbricas (OWC - Optical Wireless Communications) [11] que emplean todo tipo de dispositivos en el espectro óptico incluyendo luz infrarroja, visible y ultravioleta. Destacan en este campo las comunicaciones en espacio libre (FSO - Free-Space Optics) [12] que se encuentra en un estado de desarrollo más avanzado y las VLC, que son un tema en creciente desarrollo en los últimos años desde la masificación de las luces LED. OCC aprovecha la vasta disponibilidad de las cámaras digitales, considerablemente más abundantes que PD individuales, pero a la vez con mayores limitaciones de tasa de datos alcanzables. Por ejemplo, se han reportado sistemas VLC basados en PD que sobrepasan los Gbps de rendimiento [13], [14], en cambio, para OCC la tasa de captura de fotogramas de las cámaras convencionales supone una limitante inherente [2], [15], [16]. Se pueden encontrar así trabajos en orientados a la explotación de sistemas de modulación avanzados [17]-[19] para alcanzar mayores tasas de datos.

Tanto sistemas VLC basados en PD, como sistemas OCC basados en cámaras se han propuesto para aplicaciones de Smart Cities. Una de las que destaca es posicionamiento (VLP - Visible Light Positioning) [20]-[23]. En estos sistemas se aprovecha la alta precisión alcanzable, del orden de decenas de centímetros, comparada a sistemas de posicionamiento satelital que puede ser de decenas de metros. Los sistemas VLP tienen robustez en espacios cerrados. Estos sistemas además se relacionan con otra importante aplicación, los sistemas de transporte inteligentes utilizando VLC [4], [20], [24], [25].

Las ventajas de OCC frente a sistemas basados en PD se basan en la capacidad de las cámaras de formar imágenes y, por ende, de separar espacialmente las fuentes de luz [26][28]. Con esto se pueden mejorar sustancialmente las tasas de datos, si se emplea una topología multipunto-a-punto, como ciertos casos de redes de sensores y de Internet de las cosas (IoT - Internet of Things).

Las principales limitaciones de OCC están relacionadas con la formación de imagen y con las tasas de captura, como ya se mencionó. Entre ellas están las distorsiones causadas por la óptica [29], la sincronización entre los tiempos de captura y los de transmisión [9], [10]. Finalmente, en el procesamiento de la imagen surgen otros problemas importantes a considerar, donde destaca el descubrimiento de nodos y su seguimiento en movimiento [30].

Específicamente, sobre la implementación de sistemas subpíxel como la se propone en este artículo, los autores no conocen otros trabajos donde se reporte una funcionalidad equivalente. Cabe destacar que el escenario sub-píxel se puede considerar como un sistema VLC basado en PD, si se considera que los píxeles de las cámaras están basados en dichos dispositivos [31]. Sin embargo, como se verá en las siguientes secciones, la óptica de la cámara y la dispersión de la luz proveen oportunidad de mejorar la comunicación utilizando los demás PD presentes en la cámara.

\section{Modelo de canal en Optical Camera COMMUNICATION}

Como en cualquier sistema OWC, la potencia recibida en un sistema OCC se puede modelar empleando la aproximación de ángulo sólido diferencial (Ecuación 1) [11]. Ya que este trabajo se centra en enlaces en exteriores, se ha añadido un término de pérdidas por extinción $c(\lambda)$ en el medio (absorción y dispersión) que depende de la longitud de onda $\lambda$ [32]-[34].

$$
P_{r x}=P_{t x} R(\theta, \varphi) \frac{A_{\text {lens }}}{d^{2}} \cos (\Psi) e^{-c(\lambda) d},
$$

donde $P_{r x}$ es la potencia recibida, $P_{t x}$ es la potencia transmitida y $R(\theta, \varphi)$ es el patrón de emisión de la fuente (asumido constante en toda su área) para los ángulos de elevación $\theta$ y azimut $\varphi$. La potencia de llegada al receptor $\left(R_{x}\right)$ depende del área de la lente principal $A_{\text {lens }}$, proyectada según el ángulo de incidencia $\Psi$ y de la distancia del enlace $d$.

En OCC debe considerarse la óptica formadora de imagen de las cámaras. En términos generales y despreciando cualquier efecto de desenfoque, a priori, los sistemas OCC han estado basados en la conservación de la densidad de energía de píxel con la distancia. Esto es, la energía de cada píxel es independiente de la distancia siempre y cuando la proyección de $T_{x}$ sea superior a 1 píxel. El concepto subyacente a esta propiedad es la compensación de las pérdidas por propagación esférica con el enfoque de la imagen. Aunque llegue menos energía a la óptica principal de la cámara a medida que 
la distancia aumenta (disminución cuadrática), el número de píxeles sobre los que $T_{x}$ es proyectado también decrece con el mismo orden, manteniendo la densidad de energía constante sobre el sensor de imagen. Para largas distancias, es fácil demostrar que el número de píxeles $N_{p x}$ sobre los que se proyecta un $T_{x}$ de área $A_{t x}$ depende del campo de visión (FOV - Field of View) de la cámara y de la resolución del sensor de imagen, tal y como muestra la Ecuación 2.

$$
N_{p x}=\frac{N_{x} N_{y}}{F O V_{x} F O V_{y}} \frac{A_{t x}}{d^{2}}
$$

$N_{x}$ y $N_{y}$ definen la resolución del sensor, mientras que $F O V_{x}$ y $F O V_{y}$ definen los campos de visión horizontal y vertical de $R_{x}$ respectivamente. Uniendo las Ecuaciones $1 \mathrm{y}$ 2 , y proyectando la energía sobre el área de un píxel $\left(A_{p x}\right)$ se obtiene la Ecuación 3, la cual resume la energía promedio de un píxel.

$$
P_{p x}=P_{t x} R(\theta, \varphi) \frac{A_{l e n s}}{A_{t x}} \cos (\Psi) e^{-c(\lambda) d} \zeta_{x y} A_{p x},
$$

donde por conveniencia se ha denominado $\zeta_{x y}$ a la resolución angular del sensor denotada por el cociente entre sus FOV y la dimensión del mismo. Sin embargo, cuando la proyección de $T_{x}$ disminuye por debajo de 1 píxel, el sistema puede comenzar a modelarse como un enlace OWC basado en PD, donde la potencia recibida es directamente proporcional al área de PD iluminada por la proyección (menor a $A_{p x}$ ). El concepto mencionado se resume como sigue:

$$
P_{p x}^{s u b}=P_{p x} \cdot N_{p x}=P_{r x} \cdot A_{p x}
$$

Debe entenderse $N_{p x}$ en la Ecuación 4 como un coeficiente de ajuste referido al porcentaje de píxel iluminado. Se observa claramente que la potencia recibida en una situación sub-píxel $\left(P_{p x}^{s u b}\right)$ sí depende de la distancia y pasa a comportarse como un enlace OWC tradicional. Una vez concretada la potencia de llegada al sensor, el proceso de conversión debe tenerse en cuenta a la hora de describir la señal OCC dentro de la imagen captada. Las cámaras CMOS trabajan convirtiendo los fotones incidentes en electrones. Por tanto, es conveniente llevar a cabo una conversión de unidades que tenga en cuenta esta particularidad. En la Ecuación 5 se muestra el número de electrones almacenados $\left(E_{p x}\right)$ durante el tiempo de exposición de la captura $T_{\exp }$ (válida para cualquier situación).

$$
E_{p x}=T_{e x p} \int_{\lambda} P_{p x}(\lambda) E Q E(\lambda) \frac{E_{p h}(\lambda)}{q} \mathrm{~d} \lambda
$$

Nótese que se ha extendido el concepto de potencia de llegada al píxel incluyendo el espectro de emisión de la fuente óptica. $E Q E(\lambda)$ es la eficiencia cuántica externa (EQE External Quantum Efficiency) del sustrato del PD (silicio por lo general), $E_{p h}(\lambda)$ es la energía del fotón a cada longitud de onda y $q$ es la carga del electrón. A pesar de que teóricamente un $T_{x}$ pequeño situado a larga distancia se presentará como un único punto brillante en la captura, la óptica formadora de imagen no es perfecta, y existe cierta dispersión espacial de la energía. Esta dispersión se modela a través de la Point Spread Function (PSF) del sistema, denotada como $h[n, m]$ ya en el dominio imagen. En esencia, $h[n, m]$ es la respuesta impulsiva espacial del sistema, y depende por lo general de la distancia del enlace. Cualquier proyección debe ser convolucionada con ella, por lo que en una situación sub-píxel la región iluminada puede modelarse de la siguiente forma.

$$
s[n, m]=G_{V} \mathfrak{K}\left(E_{p x}\right) \cdot h\left[n-n_{0}, m-m_{0}\right]
$$

$\mathfrak{K}(\cdot)$ es una función que incluye la conversión analógicodigital, $G_{V}$ es la ganancia analógica de la cámara CMOS y $n_{0}$ y $m_{0}$ son las coordenadas de los píxeles donde $T_{x}$ es proyectado. Debe tenerse en cuenta que en una situación ideal, $h[n, m]$ tiene energía unitaria, por lo que si existe dispersión de energía, el nivel del píxel teórico de la proyección será menor de lo esperado.

En lo referente a la relación señal a ruido (SNR - Signalto-Noise Ratio) de un enlace OCC, la Ecuación 7 la resume, siendo aplicable tanto a situaciones sub-píxel como a las generalmente estudiadas.

$$
S N R=\frac{G_{V}^{2} E_{p x}^{2}}{G_{V}^{2}\left(\sigma_{s h}^{2}+\sigma_{t h}^{2}\right)+\sigma_{a d c}^{2}}
$$

Se ha asumido que el factor de corrección $\gamma$ de la cámara es unitario por simplicidad y sin pérdida de generalidad, así como que el enlace no está saturado (número de electrones almacenados menor que la capacidad del pozo). Las tres contribuciones principales al ruido del enlace OCC son el ruido shot $\left(\sigma_{s h}^{2}\right)$, el ruido térmico $\left(\sigma_{t h}^{2}\right)$ y el ruido de cuantificación $\left(\sigma_{a d c}^{2}\right)$. El efecto de este último puede ser minimizado aplicando el valor óptimo de ganancia analógica, tal y como se demostró en [7], [8]. Los ruidos shot se originan por la naturaleza discreta de las partículas que procesa el píxel: fotones y electrones, considerando su aparición aleatoria en el dominio del tiempo y del espacio. Dentro de los ruidos de shot, las contribuciones más significativas son el ruido de oscuridad, cuando se generan electrones sin excitación óptica, el ruido shot óptico de la propia señal generada por el fotodetector y el ruido de readout generado por los circuitos previos al conversor análogo-digital (ADC - Analog-to-Digital Converter) [31].

En los enlaces outdoor existe otro fenómeno que puede tener un impacto sustancial en el desempeño del sistema. El nivel de iluminación de fondo puede variar, al menos en una situación sub-píxel donde la velocidad viene determinada por la tasa de captura de la cámara, de manera comparable al tiempo de transmisión de una trama. Este efecto será analizado experimentalmente y discutido en la Sección V.

\section{Metodología}

El objetivo principal de este trabajo es demostrar experimentalmente la viabilidad de los enlaces OCC a larga distancia, donde la proyección de los emisores sea menor que un único píxel de la cámara. Como se ha comentado, la tecnología OCC ha sido propuesta como un habilitador para Smart Cities e IoT. Sin embargo, hasta la fecha no ha habido trabajos que validen su uso en escenarios realistas sujetos a condiciones como las que se plantean en este trabajo. A largas distancias, el uso de emisores que se proyecten en un gran número de píxeles es inviable. 

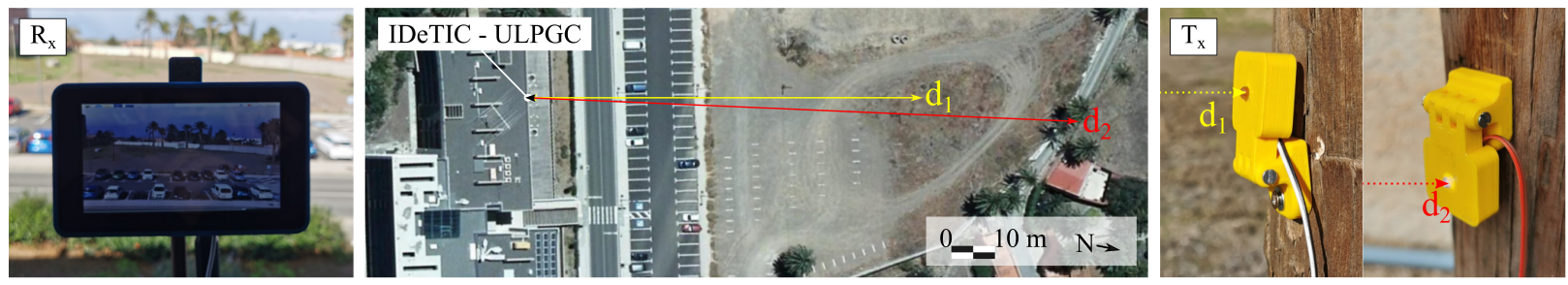

Fig. 1. Fotos de los componentes del montaje experimental incluyendo vista satelital del escenario en las inmediaciones del IDeTIC obtenida de Cartográfica de Canarias (Grafcan) [35].

Para llevar a cabo la evaluación experimental del enlace subpíxel, se diseñó un nodo $T_{x}$ OCC (Figura 1) alimentado con batería y conmutado por un microcontrolador. Se programaron dos nodos para enviar en bucle tramas de transmisión con la estructura que se muestra en la Figura 2 que contenían 1 Byte de payload en los que se transmitía secuencialmente los valores desde 0x00 hasta 0xFF. El tiempo de pulso definido para los experimentos fue de $133.33 \mathrm{~ms}$, ofreciendo una tasa binaria de 4 bps. Ambas unidades de transmisión fueron ancladas a dos postes situados a 90 metros y 130 metros de distancia de la cámara respectivamente.

Como $R_{x}$ se empleó un sensor de imagen CMOS, modelo Sony IMX219, (RaspiCam v2) controlado por una Raspberry Pi v3. Se grabó la transmisión en un vídeo de 10 minutos de duración empleando un tiempo de exposición de $85 \mu$ s a mínima ganancia analógica $(0 \mathrm{~dB})$ y sin ganancia digital. La tasa de captura de la cámara fue configurada a $30 \mathrm{fps}$ con una resolución de 1920 x 1080 px. Los parámetros experimentales se muestran en la Tabla I.

TABLA I

PARÁMETROS CLAVE DEL EXPERIMENTO

\begin{tabular}{ccc}
\hline \hline Módulo & Parámetro & Valor \\
\hline$T_{x}$ & Diámetro de $T_{x}$ & $5 \mathrm{~mm}$ \\
& Dispositivo de transmisión & 1 LED blanco estándar \\
Canal & Distancia $\left(d_{1}, d_{2}\right)$ & $90 \mathrm{~m}, 130 \mathrm{~m}$ \\
$R_{x}$ & Sensor de imagen & Sony IMX219 [36] \\
& Resolución de fotogramas & $1920 \times 1080 \mathrm{px}$ \\
& Tasa de captura & $30 \mathrm{fps}$ \\
& Tiempo de exposición $\left(t_{\text {exp }}\right)$ & $85 \mu \mathrm{s}$ \\
\hline \hline
\end{tabular}

Una vez capturado el vídeo mostrando las emisiones de ambos nodos simultáneamente, la región de interés (ROI Region of Interest) de cada transmisor se definió manualmente, ya que la elaboración de un procedimiento de descubrimiento no era objetivo de este trabajo. Ambas ROI se analizaron estadísticamente para obtener estimaciones de la SNR y de la PSF.

La PSF del sistema en ambos nodos (cercano y lejano) se estimó empleando un procedimiento basado en la correlación. Para ello se detectó el píxel de mayor energía dentro de la ROI correspondiente, y se fijó como referencia. Posteriormente se calculó la correlación de Pearson de los píxeles adyacentes respecto a la referencia. Se observó que la correlación daba valores considerablemente cercanos a la unidad para una región aproximada de $3 \times 3$ píxeles, demostrando que la imperfección del sistema óptico puede beneficiar a un enlace OCC sub-píxel. A pesar de que el efecto del background subyace al cálculo de la correlación, la matriz resultante fue asociada a la PSF como una aproximación.

Para llevar a cabo el análisis de la SNR se procesó una ventana deslizante de 150 muestras en la que se ajustó un Gaussian Mixture Model (GMM) como el de la Ecuación 8.

$$
G_{2}(x)=\frac{\alpha}{\sigma_{0} \sqrt{2 \pi}} e^{-\frac{\left(x-\mu_{0}\right)^{2}}{2 \sigma_{0}^{2}}}+\frac{1-\alpha}{\sigma_{1} \sqrt{2 \pi}} e^{-\frac{\left(x-\mu_{1}\right)^{2}}{2 \sigma_{1}^{2}}},
$$

donde $G_{2}(x)$ es una mezcla de dos Gaussianas, $\alpha$ es la proporción de la primera Gaussiana, $\mu_{i}$ denota valor esperado y $\sigma_{i}$ es desviación típica. Este procedimiento ya ha sido empleado satisfactoriamente en otros trabajos como [37]. Se empleó debido a la alta variación del nivel de background en la imagen (Figura 4), la cual podría falsear la estimación de la SNR si se tuviese en cuenta toda la señal capturada para el cálculo. La Ecuación 9 muestra cómo se llevó a cabo la estimación de la SNR.

$$
S N R=\frac{1}{2} \frac{\left|\mu_{1}-\mu_{0}\right|^{2}}{\alpha \sigma_{0}^{2}+(1-\alpha) \sigma_{1}^{2}}
$$

Usando el valor de background obtenido en cada ajuste GMM, se calculó la SNR muestra a muestra para posteriormente obtener la SNR esperada del sistema (promedio). Asumiendo que la transmisión es Non-Return to Zero On-Off Keying (NRZ-OOK), la tasa de error teórica para cada SNR estimada experimentalmente se calculó empleando la Ecuación 10.

$$
B E R=\frac{1}{2} \operatorname{erfc}\left(\sqrt{\frac{S N R}{2}}\right),
$$

donde $\operatorname{erfc}(\cdot)$ es la función de error complementario. Tanto el cálculo de la SNR como de la BER fue llevado a cabo para cada color del sensor de imagen (R, G y B) y para tres esquemas de detección: píxel de máxima energía, media (Ecuación 11) y media enfatizada con la aproximación de la PSF (Ecuación 12). El objetivo del promediado espacial es mejorar la SNR al reducir la varianza efectiva de ruido.

$$
\begin{gathered}
y_{\text {mean }}=\frac{1}{N M} \sum_{i=0}^{N-1} \sum_{j=0}^{M-1} x[i, j] \\
y_{\text {emph }}=\frac{\sum_{i=0}^{N-1} \sum_{j=0}^{M-1} \rho[i, j] \cdot x[i, j]}{\sum_{i=0}^{N-1} \sum_{j=0}^{M-1} \rho[i, j]}
\end{gathered}
$$

$N$ y $M$ son el alto y ancho de la ROI considerada para el promediado, $y_{\text {mean }}$ es la señal resultante de la media aritmética, $y_{\text {emph }}$ es la señal resultantes del promediado enfatizado, $s[i, j]$ es la señal original en la coordenada $(i, j)$ y $\rho[i, j]$ es la correlación descrita anteriormente. 


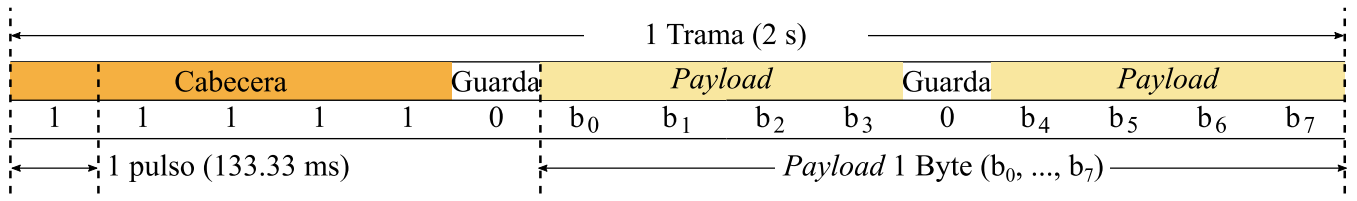

Fig. 2. Formato de trama empleado en el experimento.

\section{IV-A. Algoritmo de detección OCC}

Además de estimar la BER teórica, se implementó un algoritmo de detección basado en correladores, uno por cada posible trama enviada (256 en total). El algoritmo lleva a cabo una multiplicación matriz-vector con cada muestra adquirida, para posteriormente encontrar el índice asociado al valor máximo. La matriz de correladores, $\mathbf{S}$, se formó a partir de la estandarización de los vectores asociados a cada una de las tramas posibles (Ecuación 13).

$$
\hat{\mathbf{s}}_{\mathbf{i}}=\frac{N \mathbf{s}_{\mathbf{i}}-\left\|\mathbf{s}_{\mathbf{i}}\right\|_{L_{0}}}{\sqrt{\left(\left\|\mathbf{s}_{\mathbf{i}} \mid\right\|_{L_{0}}\right)\left(N-\left\|\mathbf{s}_{\mathbf{i}}\right\|_{L_{0}}\right)}}
$$

donde $\hat{s}_{\mathbf{i}}$ es el vector de la i-ésima trama, sometido a estandarización estadística, que fuerza la distribución a una de media y desviación típica igual a 0 y a 1 , respectivamente. $\mathbf{s}_{\mathbf{i}}$ es el vector original, $N$ es el tamaño de la trama, el cual en este trabajo es 60 acorde al diseño de la trama y a la frecuencia de muestreo y $\|\cdot\|_{L_{0}}$ es la norma $L_{0}$ (número de elementos no nulos del vector). Al llevar a cabo la correlación de esta manera, únicamente debe hacerse una multiplicación matriz-vector de orden 256x60, la cual tiene un consumo computacional despreciable. Para que el resultado de la multiplicación se corresponda con la correlación de Pearson y poder definir umbrales en el proceso de sincronización, el buffer de longitud 60 sobre el que se aplica el producto es también sometido a estandarización estadística acorde a la Ecuación 14.

$$
\hat{\mathbf{y}}=\frac{\mathbf{y}-\overline{\mathbf{y}}}{\sigma_{y}}
$$

$\mathrm{Al}$ igual que en el caso anterior, $\hat{\mathbf{y}}$ es el buffer de señal de entrada normalizado, $\mathbf{y}$ es el original, $\overline{\mathbf{y}}$ es la media aritmética y $\sigma_{y}$ la desviación típica. Acorde a la definición de la correlación de Pearson, al hacer el producto $\mathbf{S} \cdot \hat{\mathbf{y}}$ se obtiene un vector de correlaciones $\mathbf{c}$ que puede ser empleado para detectar el símbolo transmitido. La Figura 3 ilustra el proceso de detección.

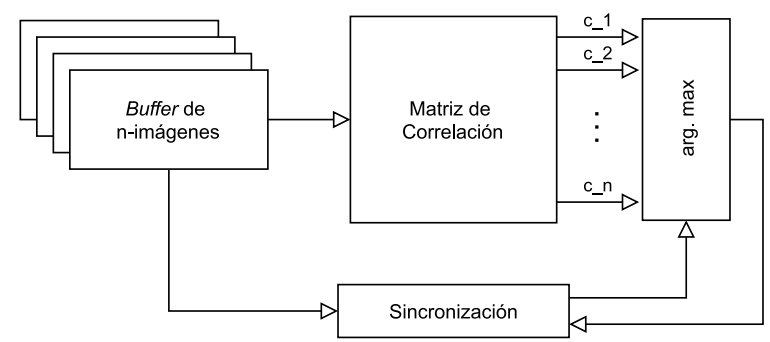

Fig. 3. Diagrama de flujo de $R_{x}$

El esquema de sincronización empleado ha sido el siguiente. Una vez se llena el buffer, se espera a la primera trama que supere la correlación umbral, $c_{t h}$ de 0.94 . Este valor fue escogido de manera empírica, usando los procedimientos desarrollados en [7], [8]. El Byte transmitido se decodifica directamente buscando el argumento máximo del vector c. Tras dicha detección, se inhibe el proceso de decisión durante las siguientes 55 muestras y posteriormente se almacenan pares correlación-Byte decodificado para las siguientes 10 muestras. Dentro de ese buffer secundario se hace la búsqueda del instante de mayor correlación y se asume que el Byte asociado se corresponde a aquel con mayor verosimilitud. El número de muestras descartadas a partir de este momento será 50 más el índice del valor de máxima correlación encontrado en el buffer secundario.

\section{Resultados}

Como se comentó anteriormente, se capturó un vídeo de 10 minutos en el que dos nodos OCC situados a dos distancias diferentes estuvieron enviando de manera consecutiva información desde el Byte 0x00 hasta el Byte 0xFF. La Figura 4 muestra, a modo de ejemplo, la forma de onda capturada para ambos nodos en el píxel de máxima energía. Como puede observarse, el nivel de background varió considerablemente durante la transmisión, además de la ganancia del canal, visible en la amplitud variable de la señal recibida.

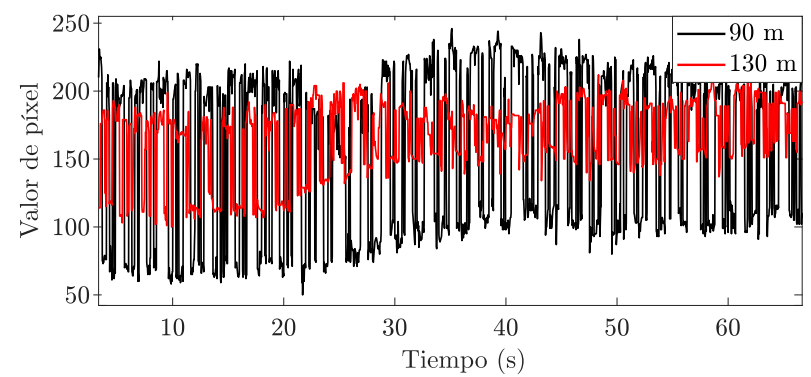

Fig. 4. Ejemplo de forma de onda capturada en el píxel de máxima energía para los dos nodos desplegados.

$\mathrm{Al}$ estar en una situación sub-píxel en la que el FOV de $R_{x}$ es tan reducido (orden de miliestereoradianes), cualquier perturbación atmosférica o vibración que afecte al alineamiento puede tener un impacto en el enlace. Un ejemplo de imagen capturada con ambos nodos resaltados se muestra en la Figura 5. Este resultado demuestra que la pequeña dimensión de $T_{x}$ y el amplio campo de visión de $R_{x}$ permitirían el uso de OCC para la monitorización de una gran cantidad de sensores (varias centenas) de manera simultánea, explotando la capacidad de multiplexación espacial inherentes a las cámaras.

El procedimiento de aproximación de la PSF de los enlaces se aplicó a las señales capturadas en color convertidas a escala de grises (luminancia), obteniendo los resultados de la 




Fig. 5. Ejemplo de imagen capturada en el entorno de pruebas en exteriores. Las imágenes incrustadas en círculos muestran los estados encendido y apagado de ambos nodos.

Figura 6. Aunque la proyección de los nodos $T_{x}(5 \mathrm{~mm}$ de diámetro) sobre el sensor de imagen es menor a un único píxel, se observa que existe una región en la cual la correlación es suficiente como para asegurar que existe señal. Esta dispersión en la energía ocurre debido a la imposibilidad de enfocar por completo toda la energía proveniente de un cierto ángulo sólido en un único píxel. Además, la PSF del enlace a 90 metros tiene mayor dispersión que la PSF a 130 metros, posiblemente debido a la distancia focal fijada durante la captura de los vídeos, así como a las imperfecciones del sistema de lentes.
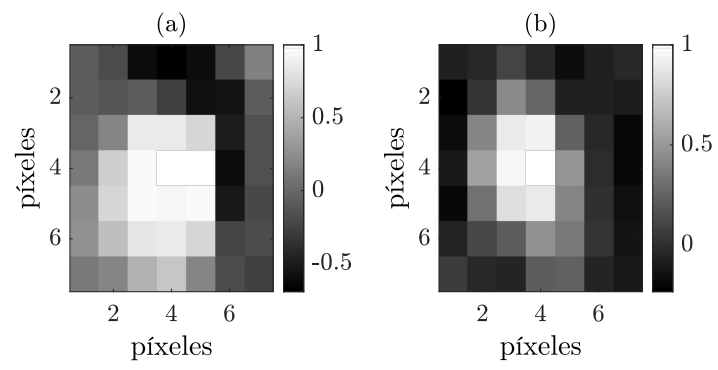

Fig. 6. Estimación de la PSF a partir de la correlación respecto al píxel de mayor energía. (a) PSF estimada a 90 metros. (b) PSF estimada a 130 metros.

La Figura 7 muestra las formas de onda asociadas a los píxeles $(4,4)$ y $(3,4)$ de la PSF para el enlace lejano. A medida que el píxel observado se aleja del de máxima energía, la correlación se pierde y la onda asociada al píxel queda únicamente definida por la variación de la iluminación de fondo de la imagen.

(a)



Fig. 7. Formas de onda para el nodo lejano (130 metros). (a) Píxel de máxima energía o $(4,4)$ de la PSF. (b) Píxel $(3,4)$ de la PSF.
Como se explicó en la Sección IV, se obtuvieron la SNR y la BER de ambos nodos, tanto teórica como experimental y empleando diferentes esquemas de promediado espacial. La Tabla II muestra los resultados para el esquema de detección basado en 1 píxel (de máxima energía), mientras que la Tabla III muestra los resultados para el esquema basado en la media de todos los píxeles de la región de la PSF y la Tabla IV muestra los resultados usando el esquema enfatizado por la PSF.

El nodo cercano alcanzó una SNR experimental máxima de $20 \mathrm{~dB}$ en el canal verde ( $\mathrm{G}$ - Green) utilizando el método de promediado enfatizado usando la PSF estimada. Su peor valor de SNR alcanzado fue de $17,1 \mathrm{~dB}$ para el canal rojo ( $\mathrm{R}$ - Red) usando el método de cálculo basado sólo en el píxel de mayor correlación en la PSF. La BER fue estimada teóricamente como inferior a $10^{-12}$, dados los altos valores de SNR. En el experimento no se encontraron errores con ninguno de los métodos de promediado (el $*$ indica que la BER es menor a $3.33 \cdot 10^{-3}$ ). Esto se debe a que no se ejecutó un número suficiente de experimentos.

En el caso del nodo lejano, donde la proyección del píxel en el sensor es comparablemente menor, la SNR fluctuó entre $8,1 \mathrm{~dB}$ y $13 \mathrm{~dB}$, para el canal $\mathrm{G}$ en esquema 1-píxel y el $R$ en esquema enfatizado, respectivamente. La BER teórica estimada fue del orden de $10^{-3}, 10^{-5}$ y $10^{-6}$ para los esquemas 1-píxel, promediado y enfatizado, respectivamente. En este caso los valores de BER son más altos y por lo tanto, hacen falta menos experimentos por ejecutar. Las BER experimentales obtenidas para este experimento discreparon bastante de los niveles teóricos, siendo por ejemplo, una diferencia de 1 hasta 3 órdenes de magnitud. Ésto puede deberse a la naturaleza no-estacionaria del canal, que afecta más en este caso de baja SNR, relativamente, y que no es superada de manera efectiva por el sistema de detección.

TABLA II

MÉTRICAS EN ESQUEMA 1-PÍXEL

\begin{tabular}{ccccc}
\hline \hline Métrica & Posición & Canal R & Canal G & Canal B \\
\hline SNR exp. & $d_{1}$ & $17,1 \mathrm{~dB}$ & $17,3 \mathrm{~dB}$ & $17,3 \mathrm{~dB}$ \\
SNR exp. & $d_{2}$ & $8,1 \mathrm{~dB}$ & $8,1 \mathrm{~dB}$ & $8,2 \mathrm{~dB}$ \\
BER teórica & $d_{1}$ & $<10^{-12}$ & $<10^{-12}$ & $<10^{-12}$ \\
BER teórica & $d_{2}$ & $5,50 \cdot 10^{-3}$ & $5,50 \cdot 10^{-3}$ & $5,10 \cdot 10^{-3}$ \\
BER exp. & $d_{1}$ & $0^{*}$ & $0^{*}$ & $0^{*}$ \\
BER exp. & $d_{2}$ & $2,22 \cdot 10^{-2}$ & $2,22 \cdot 10^{-2}$ & $8,9 \cdot 10^{-3}$ \\
\hline \hline
\end{tabular}

TABLA III

MÉTRICAS EN ESQUEMA PROMEDIADO

\begin{tabular}{ccccc}
\hline \hline Métrica & Posición & Canal R & Canal G & Canal B \\
\hline SNR exp. & $d_{1}$ & $19,6 \mathrm{~dB}$ & $19,9 \mathrm{~dB}$ & $19,7 \mathrm{~dB}$ \\
SNR exp. & $d_{2}$ & $12,6 \mathrm{~dB}$ & $12,3 \mathrm{~dB}$ & $12,3 \mathrm{~dB}$ \\
BER teórica & $d_{1}$ & $<10^{-12}$ & $<10^{-12}$ & $<10^{-12}$ \\
BER teórica & $d_{2}$ & $9,96 \cdot 10^{-6}$ & $1,88 \cdot 10^{-5}$ & $1,88 \cdot 10^{-5}$ \\
BER exp. & $d_{1}$ & $0^{*}$ & $0^{*}$ & $0^{*}$ \\
BER exp. & $d_{2}$ & $1,20 \cdot 10^{-2}$ & $1,20 \cdot 10^{-2}$ & $9,60 \cdot 10^{-3}$ \\
\hline \hline
\end{tabular}

\section{Conclusiones}

El objetivo fundamental de este trabajo es demostrar la viabilidad de los enlaces OCC sub-píxel a través de una 
TABLA IV

MÉTRICAS EN ESQUEMA ENFATIZADO

\begin{tabular}{ccccc}
\hline \hline Métrica & Posición & Canal R & Canal G & Canal B \\
\hline SNR exp. & $d_{1}$ & $19,7 \mathrm{~dB}$ & $20,0 \mathrm{~dB}$ & $19,8 \mathrm{~dB}$ \\
SNR exp. & $d_{2}$ & $13,0 \mathrm{~dB}$ & $12,9 \mathrm{~dB}$ & $12,5 \mathrm{~dB}$ \\
BER teórica & $d_{1}$ & $<10^{-12}$ & $<10^{-12}$ & $<10^{-12}$ \\
BER teórica & $d_{2}$ & $3,97 \cdot 10^{-6}$ & $5,03 \cdot 10^{-6}$ & $1,24 \cdot 10^{-5}$ \\
BER exp. & $d_{1}$ & $0^{*}$ & $0^{*}$ & $0^{*}$ \\
BER exp. & $d_{2}$ & $9,60 \cdot 10^{-3}$ & $9,60 \cdot 10^{-3}$ & $7,2 \cdot 10^{-3}$ \\
\hline \hline
\end{tabular}

validación experimental en un escenario realista. Una de las promesas recurrentes de esta tecnología es la posibilidad de usar cámaras en Smart Cities para aplicaciones de monitorización masiva de sensores. Sin embargo, hasta ahora los sistemas OCC se han basado en el uso de lámparas de gran tamaño, complementadas con óptica de alta ganancia (objetivos de alta distancia focal), para poder hacer uso de las técnicas RS. No obstante, en los casos de uso que se proponen en este trabajo, la velocidad de transmisión es un factor no crítico, por lo que se permite la explotación de la inherente capacidad de multiplexación espacial de las cámaras.

Para validar la hipótesis de partida, se diseñó un sistema experimental en que la proyección de cada nodo emisor $\left(T_{x}\right)$ sobre el sensor de imagen fuese de menor tamaño que un único píxel. Se construyeron dos nodos transmisores alimentados con batería, transmitiendo una secuencia fácilmente decodificable modulada usando NRZ-OOK y un nodo $R_{x}$ basado en una cámara comercial situada a larga distancia. Se probaron dos enlances a distancias de $90 \mathrm{~m}$ y $130 \mathrm{~m}$.

El sistema alcanzó una SNR relativamente igual para los canales rojo, verde y azul, con valores de aproximadamente $17 \mathrm{~dB}$ y $8 \mathrm{~dB}$ para $90 \mathrm{~m}$ y $130 \mathrm{~m}$, respectivamente. Usando el mejoramiento por PSF enfatizado se incrementaron estos valores a $20 \mathrm{~dB}$ y $13 \mathrm{~dB}$. Considerando que se utilizó la técnica NRZ-OOK se estimó la cota teórica de la BER para cada distancia y se puede observar que la BER experimental dista considerablemente para el caso $d_{2}$ dado que el canal es no estacionario. Para el caso $d_{1}$ no se encontró ningún error durante los experimentos.

El esquema de comunicación presentado en este trabajo puede escalarse mediante el aumento de nodos transmisores y el uso de mayores velocidades de captura de fotogramas, alcanzando tasas de bits del orden de decenas de kbps compartida entre los nodos. Las características que se muestran en este trabajo hacen a los sistemas OCC sub-píxel particularmente atractivos para su aplicación en Smart Cities como por ejemplo en la monitorización de sensores en entornos industriales, a distancias de varias decenas de metros y cargando datos a baja velocidad a un nodo central basado en una cámara comercial que podría ser simultáneamente un equipo de vigilancia, dado que los fotogramas contienen tanto los datos como la imagen del entorno. La eficiencia energética de los LED y la implementación simple de la modulación NRZ-OOK reducen la complejidad y consumo de los nodos transmisores, que en redes de sensores están limitados energéticamente. Además, este tipo de esquemas podría utilizarse en interiores, a pequeñas distancias, aprovechando luces micro-LED aún más bajas en consumo, para aplicaciones IoT de monitorización de máquinas en entornos de hogar, oficina.

\section{REFERENCIAS}

[1] IEEE Standard Association, "IEEE standard for local and metropolitan area networks-part 15.7: short-range wireless optical communication using visible light," IEEE: Piscataway, NZ, USA, pp. 1-309, 2011.

[2] N. Saeed, S. Guo, K.-H. Park, T. Y. Al-Naffouri, and M.-S. Alouini, "Optical camera communications: Survey, use cases, challenges, and future trends," Physical Communication, vol. 37, p. 100900, 2019.

[3] E. Eso, S. Teli, N. B. Hassan, S. Vitek, Z. Ghassemlooy, and S. Zvanovec, "400 m rolling-shutter-based optical camera communications link," Opt. Lett., vol. 45, pp. 1059-1062, Feb 2020.

[4] M. Karbalayghareh, F. Miramirkhani, H. B. Eldeeb, R. C. Kizilirmak, S. M. Sait, and M. Uysal, "Channel modelling and performance limits of vehicular visible light communication systems," IEEE Transactions on Vehicular Technology, vol. 69, no. 7, pp. 6891-6901, 2020.

[5] P. Chavez-Burbano, V. Guerra, J. Rabadan, and R. Perez-Jimenez, "Optical camera communication for smart cities," in 2017 IEEE/CIC International Conference on Communications in China (ICCC Workshops), pp. 1-4, 2017.

[6] Y. H. Kim, W. A. Cahyadi, and Y. H. Chung, "Experimental demonstration of vlc-based vehicle-to-vehicle communications under fog conditions," IEEE Photonics Journal, vol. 7, no. 6, pp. 1-9, 2015.

[7] V. Matus, V. Guerra, C. Jurado-Verdu, S. Teli, S. Zvanovec, J. Rabadan, and R. Perez-Jimenez, "Experimental evaluation of an analog gain optimization algorithm in optical camera communications," in 2020 12th International Symposium on Communication Systems, Networks Digital Signal Processing (CSNDSP), pp. 1-5, 2020.

[8] V. Matus, E. Eso, S. R. Teli, R. Perez-Jimenez, and S. Zvanovec, "Experimentally derived feasibility of optical camera communications under turbulence and fog conditions," Sensors, vol. 20, p. 757, Jan 2020.

[9] C. Jurado-Verdu, V. Matus, J. Rabadan, V. Guerra, and R. Perez-Jimenez, "Correlation-based receiver for optical camera communications," Opt. Express, vol. 27, pp. 19150-19155, Jul 2019.

[10] C. Jurado-Verdu, V. Guerra, J. Rabadan, R. Perez-Jimenez, and P. Chavez-Burbano, "Rgb synchronous vlc modulation scheme for occ," in 2018 11th International Symposium on Communication Systems, Networks Digital Signal Processing (CSNDSP), pp. 1-6, July 2018.

[11] Z. Ghassemlooy, W. Popoola, and S. Rajbhandari, Optical wireless communications: system and channel modelling with Matlab. CRC press, 2019.

[12] M. A. Khalighi and M. Uysal, "Survey on free space optical communication: A communication theory perspective," IEEE Communications Surveys Tutorials, vol. 16, no. 4, pp. 2231-2258, 2014.

[13] P. H. Pathak, X. Feng, P. Hu, and P. Mohapatra, "Visible light communication, networking, and sensing: A survey, potential and challenges," IEEE communications surveys \& tutorials, vol. 17, no. 4, pp. 2047-2077, 2015.

[14] Y. Almadani, D. Plets, S. Bastiaens, W. Joseph, M. Ijaz, Z. Ghassemlooy, and S. Rajbhandari, "Visible light communications for industrial applications-challenges and potentials," Electronics, vol. 9, no. 12, 2020.

[15] N. Saha, M. S. Ifthekhar, N. T. Le, and Y. M. Jang, "Survey on optical camera communications: challenges and opportunities," IET Optoelectronics, vol. 9, pp. 172-183(11), October 2015.

[16] N. T. Le, M. Hossain, and Y. M. Jang, "A survey of design and implementation for optical camera communication," Signal Processing: Image Communication, vol. 53, pp. 95-109, 2017.

[17] J. L. H. Rios, "Experimental validation of inverse mppm modulation for dimming control and data transmission in visible light communications," IEEE Latin America Transactions, vol. 100, no. 1e, 2020.

[18] O. I. Younus, N. Bani Hassan, Z. Ghassemlooy, P. A. Haigh, S. Zvanovec, L. N. Alves, and H. L. Minh, "Data rate enhancement in optical camera communications using an artificial neural network equaliser," IEEE Access, vol. 8, pp. 42656-42665, 2020.

[19] P. Haigh, P. Chvojka, Z. Ghassemlooy, S. Zvanovec, and I. Darwazeh, "Visible light communications: multi-band super-nyquist cap modulation," Optics express, vol. 27, no. 6, pp. 8912-8919, 2019.

[20] A. L. R. Gonçalves, Á. H. A. Maia, M. R. Santos, D. A. de Lima, and A. de Miranda Neto, "Visible light positioning and communication methods and their applications in the intelligent mobility," IEEE Latin America Transactions, vol. 100, no. 1e, 2021.

[21] N. Chaudhary, O. I. Younus, L. N. Alves, Z. Ghassemlooy, S. Zvanovec, and H. Le-Minh, "An indoor visible light positioning system using tilted leds with high accuracy," Sensors, vol. 21, no. 3, 2021. 
[22] P. Palacios Játiva, M. Román Cañizares, C. A. Azurdia-Meza, D. ZabalaBlanco, A. Dehghan Firoozabadi, F. Seguel, S. Montejo-Sánchez, and I. Soto, "Interference mitigation for visible light communications in underground mines using angle diversity receivers," Sensors, vol. 20, no. $2,2020$.

[23] D. Iturralde, C. Azurdia-Meza, N. Krommenacker, I. Soto, Z. Ghassemlooy, and N. Becerra, "A new location system for an underground mining environment using visible light communications," in 2014 9th International Symposium on Communication Systems, Networks \& Digital Sign (CSNDSP), pp. 1165-1169, IEEE, 2014.

[24] R. M. Marè, C. L. Marte, C. E. Cugnasca, O. G. Sobrinho, and A. S. dos Santos, "Feasibility of a testing methodology for visible light communication systems applied to intelligent transport systems," IEEE Latin America Transactions, vol. 100, no. 1e, 2020.

[25] M. Elamassie, M. Karbalayghareh, F. Miramirkhani, R. C. Kizilirmak, and M. Uysal, "Effect of fog and rain on the performance of vehicular visible light communications," in 2018 IEEE 87th Vehicular Technology Conference (VTC Spring), pp. 1-6, 2018.

[26] S. R. Teli, S. Zvanovec, R. Perez-Jimenez, and Z. Ghassemlooy, "Spatial frequency-based angular behavior of a short-range flicker-free mimo-occ link," Appl. Opt., vol. 59, pp. 10357-10368, Nov 2020.

[27] S. R. Teli, V. Matus, S. Zvanovec, R. Perez-Jimenez, S. Vitek, and Z. Ghassemlooy, "The first study of mimo scheme within rolling-shutter based optical camera communications," in 2020 12th International Symposium on Communication Systems, Networks and Digital Signal Processing (CSNDSP), pp. 1-5, 2020.

[28] Nam-Tuan Le and Yeong Min Jang, "Performance evaluation of mimo optical camera communications based rolling shutter image sensor," in 2016 Eighth International Conference on Ubiquitous and Future Networks (ICUFN), pp. 140-144, 2016.

[29] A. Ashok, S. Jain, M. Gruteser, N. Mandayam, W. Yuan, and K. Dana, "Capacity of pervasive camera based communication under perspective distortions," in 2014 IEEE International Conference on Pervasive Computing and Communications (PerCom), pp. 112-120, IEEE, 2014.

[30] J. Shi, J. He, Z. Jiang, Y. Zhou, and Y. Xiao, "Enabling user mobility for optical camera communication using mobile phone," Optics express, vol. 26, no. 17, pp. 21762-21767, 2018.

[31] T. Kuroda, Essential Principles of Image Sensors. CRC Press, 2017.

[32] A. Ishimaru, Electromagnetic wave propagation, radiation, and scattering: from fundamentals to applications. John Wiley \& Sons, 2017.

[33] D. Kedar and S. Arnon, "Urban optical wireless communication networks: The main challenges and possible solutions," Communications Magazine, IEEE, vol. 42, pp. S2 - S7, 062004.

[34] D. Kedar and S. Arnon, "The positive contribution of fog to the mitigation of pointing errors in optical wireless communication," Applied Optics, vol. 42, no. 24, pp. 4946-4954, 2003.

[35] Cartográfica de Canarias (GRAFCAN), "Sistema de información territorial de Canarias."

[36] Sony Corporation, IMX219PQH5-C, Diagonal 4.60 mm (Type 1/4.0) 8 Mega-Pixel CMOS Image Sensor with Square Pixel for Color Cameras, Datasheet. Sony Corporation, 2014.

[37] V. Matus, V. Guerra, S. Zvanovec, J. Rabadan, and R. Perez-Jimenez, "Sandstorm effect on experimental optical camera communication," Appl. Opt., vol. 60, pp. 75-82, Jan 2021.

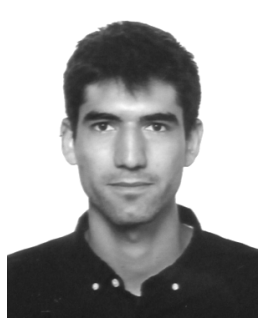

Vicente Matus obtuvo el grado de Ingeniero Eléctrico en 2018 en la Universidad de Chile. En la actualidad es un becario de doctorado en el proyecto Marie Skłodowska-Curie Innovative Training Network VisIoN (G.A. 764461) en Instituto Universitario IDeTIC de la ULPGC, España. Su campo de investigación se enfoca en las comunicaciones ópticas mediante cámaras y sus aplicaciones en redes de sensores.

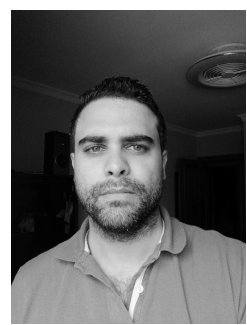

Victor Guerra MEng. en Telecomunicaciones (ULPGC, 2010), MSc. en Sistemas Inteligentes (ULPGC, 2012) y PhD (ULPGC, 2016). Premio Extraordinario de Doctorado en la Rama de Ingeniería y Arquitectura (ULPGC, 2018). Investigador de la División de Fotónica y Comunicaciones del Instituto Universitario IDeTIC desde 2008. Ha participado en 4 proyectos $\mathrm{H} 2020,3$ proyectos nacionales y 2 de carácter regional, además de en varios contratos con empresas privadas de alta relevancia. Ha publicado más de 50 artículos en revistas indexadas y comunicaciones en congresos. Sus intereses actuales de investigación son las comunicaciones ópticas inalámbricas (atmosféricas y submarinas), las comunicaciones ópticas por cámara y las aplicaciones de inteligencia artificial.

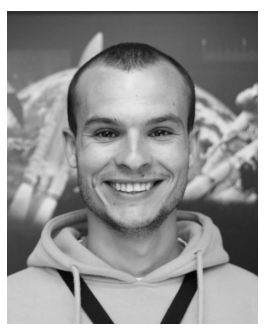

Cristo Jurado-Verdu obtuvo el máster en Ingeniería en Tecnologías de Telecomunicación en la ULPGC en 2019, España. Actualmente, está realizando su doctorado en comunicaciones ópticas inalámbricas en el Instituto Universitario IDeTIC de la ULPGC. Su investigación está enfocada al desarrollo de potenciales aplicaciones de las comunicaciones ópticas basadas en cámara. Sus intereses están relacionados con la optimización de firmware embebido, la automatización industrial, y la implementación de aplicaciones de visión artificial.

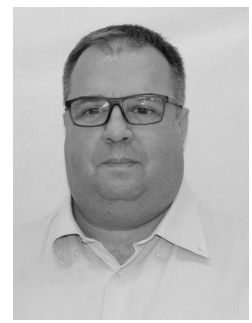

Jose Rabadan es Ingeniero de Telecomunicación (1995) y Doctor (2000) por la Universidad de Las Palmas de Gran Canaria, donde actualmente es profesor e investigador adscrito al Instituto Universitario IDeTIC. Sus intereses de investigación se enmarcan en el campo de las comunicaciones ópticas inalámbricas, en los sistemas de comunicación por luz visible y comunicaciones ópticas con cámaras, donde trabaja en esquemas de codificación y modulación de alto rendimiento y en técnicas de estimación de canales. Ha sido investigador en diferentes proyectos nacionales e internacionales financiados por administraciones y empresas nacionales y europeas. También es autor de 3 capítulos de libros, más de 30 artículos en revistas internacionales y más de 90 ponencias en congresos.



Rafael Perez-Jimenez Madrid 1965. Eng. y MSc. (UPM, 1991), PhD (ULPGC 1995 y ULL en 2020), catedrático en la ULPGC desde 2003, Director del Instituto Universitario IDeTIC de la ULPGC entre 2008 y 2020. Ha participado en 9 proyectos de investigación internacionales, más de 20 proyectos nacionales y una veintena de contratos relevantes con empresas y administraciones. Ha publicado 4 libros docentes, 5 capítulos de libro y más de 250 artículos, comunicaciones y ponencias en congresos internacionales. Su área de especialización corresponde al desarrollo de sistemas de comunicaciones ópticas para redes de sensores y enlaces de media/baja velocidad, y a la caracterización de canales ópticos en interiores y en sistemas móviles. Actualmente es coordinador dentro del área de Tecnología de la Información y Comunicaciones de la Agencia Estatal de Investigación de España. 